\title{
An outside-option-sensitive allocation rule for networks: the kappa-value
}

\author{
Julia Belau
}

Received: 23 September 2013 / Accepted: 10 October 2013 / Published online: 23 October 2013 (C) SAET 2013

\begin{abstract}
How should we allocate worth among agents in cooperative models? The literature provides various examples suggesting that an allocation rule should take outside options into account (bargaining power, etc.) as well as the specific position of agents within the network describing the social or economic structure (trade, social networks, etc.). Existing allocation rules lack at least one of these properties, either generally or for a broad and important class of games. We define and characterize a new allocation rule for networks, the kappa-value, which takes into account both outside options and the position of the agents within the network. The characterization of the kappa-value only consists of known and approved axioms or weakened versions of them and provides an elegant use of the quite intuitive concept of the Shapley value of the arc game, the Position value, lacking its drawback of outside-option-insensitivity.
\end{abstract}

Keywords Cooperative games $\cdot$ Networks $\cdot$ Position value $\cdot$ Outside options

\section{JEL Classification C71 $\cdot$ D85}

\section{Introduction}

In an economic or social situation where agents have to group in order to achieve common goals, how should we allocate the worth arising from the coalition formation among the agents? There are various frameworks and various allocation rules. This paper suggests an allocation rule for network structures which, in contrast to existing

\footnotetext{
J. Belau $(\varangle)$

Department of Economics and Social Science, TU Dortmund University and

Ruhr Graduate School in Economics,

44227 Dortmund, Germany

e-mail: julia.belau@tu-dortmund.de
} 
allocation rules, accounts for both outside options and the role of an agent within the network.

We analyze situations where coalition formation is restricted by an undirected graph, that is, the economic or social structure is described by a network which captures the (bilateral) relations between agents and agents can only form a coalition if they are connected in such an interaction network via path (directly or indirectly). This approach was introduced by Myerson (1977) [for applications and further approaches on communication structures, see for example, van den Brink et al. (2007) or van den Brink et al. (2011)]. We use undirected networks to describe the relations between the agents while it would also be possible to use directed networks (i.e., interaction has a fixed direction) as analyzed by González-Arangüena et al. (2008). They use generalized characteristic functions as introduced by Nowak and Radzik (1994), that is, games where the worth of a coalition depends on the order in which agents enter the coalition. In our approach, interaction is not directed and the order of entry does not change the worth of the coalition.

There are various examples in which an allocation rule should take into account outside options. As a simple example, consider a glove game, introduced by Shapley and Shubik (1969), with two right gloves and one left glove. A pair of gloves produces worth which has to be distributed among the agents holding the gloves. Due to the bargaining position (outside options) of the left glove holder, this agent should obtain a higher payoff than the others. This game has a nice economic interpretation and it is used to analyze simple markets (cf. Shapley and Shubik 1969). Tutic et al. (2011) show in their experimental work about social interchange that outside options significantly affect negotiations. This connection has also been mentioned by Maschler (1992) as "the need to let the players know what to expect from each coalition structure so that they can then make up their mind about the coalitions they want to join, and in what configuration" (p. 595). In a similar spirit, von Neumann and Morgenstern (1944) state that any formed coalition between individuals "only describes one particular consideration". The result of negotiation between the individuals will be "decisively influenced by the other alliances which each one might alternatively have entered". "Even if [...] one particular alliance is actually formed, the others are present in virtual existence: although they have not materialized, they have contributed essentially to shaping and determining the actual reality." (p. 36)

Furthermore, an allocation rule should take into account the specific position of agents within the network, that is, taking into account the path of information flow. As a simple example take the train connections between England, France and Germany. The Eurostar train connects London and Paris and the Thalys train connects Paris and Cologne while there is no direct train connection between England and Germany. Hence, as a transit country, France has to be passed for any kind of flow via trains between England and Germany (travel, trade,...). As further examples consider cost allocation among the nodes in energy networks or social networks used for job offers: nodes with a lot of links should be treated differently to nodes with just a few links. 
The literature suggests different allocation rules. The Shapley value (Shapley 1953) as well as the component-restricted Shapley value ${ }^{1}$ (Aumann and Drèze 1974) and, as another modification of the Shapley value, the Owen value (Owen 1977) do not take into account the network structure as they are designed for coalitional models without any interaction structure. Also more recent allocation rules that account for outside options, the Wiese value (Wiese 2007) and the $\chi$-value (Casajus 2009b), do not account for the inner interaction structure of a coalition. In order to account for the position within the network, this paper only analyzes allocation rules designed for networks (without further structure). These are the Position value (Meessen 1988; Borm et al. 1992), the graph- $\chi$-value (Casajus 2009a) and the Myerson value (Myerson 1977) or, as a generalization of the latter to a wider class of networks, the equal bargaining rule (Jackson and Wolinsky 1996). ${ }^{2}$ The Position value never accounts for structures outside the own coalition, hence, is outside-option-insensitive while the graph- $\chi$ value generally does not take into account the network structure within a coalition for weighted voting games with minimal winning coalitions. The Myerson value, and hence the equal bargaining rule, even have both these drawbacks. Hence, there is a need for a new allocation rule.

The contribution of this paper is the definition and characterization of a new allocation rule, the kappa-value, that is outside-option-sensitive and takes into account the position of an agent within the network. To the best of our knowledge, there exists no other allocation rule combining these properties. The kappa-value has a nice axiomatization, we only need already known and approved axioms or weakened versions of them (which combine the ideas of known and approved axioms). The kappa-value combines the advantages of the graph- $\chi$-value and the Position value while lacking their drawbacks and furthermore provides an elegant use of the quite intuitive concept of the Position value. We further show the independence of the characterizing axioms.

To motivate the need for an allocation rule accounting for both outside options and the specific position of agents within the network, consider the following weighted voting game: we have four agents $\{1,2,3,4\}$ holding weights $\left(w_{1}, w_{2}, w_{3}, w_{4}\right)=$ $(39,30,25,6)$ and let the threshold be $T=60$, that is, the worth of a coalition is 1 if the sum of weights of the coalitional agents is at least 60 and 0 otherwise. Hence, minimal winning coalitions are $\{1,2\},\{1,3\}$ and $\{2,3,4\}$. We consider minimal winning coalitions without organized opposition, that is, agents outside the winning coalition stay as singletons. The networks that could occur are shown in Fig. 1.

Table 1 reports the distribution of worth assigned by the Position value, denoted by $\pi$, the graph- $\chi$-value, denoted by $\chi^{\#}$ and the new allocation rule suggested in this paper, the kappa-value, denoted by $\kappa .^{3}$

Consider the networks $g^{3-2-4}$ and $g^{2-3-4}$ : the graph- $\chi$-value assigns the same payoff to agent 2 even though its position in the first case is stronger than in the

\footnotetext{
1 Also known as AD value.

2 We do not analyze the equal splitting rule (Jackson and Wolinsky 1996), since it does not account for any inner structure (neither coalitions nor interaction between agents).

3 Note that the value function considers all possible winning coalitions, not only the minimal ones. We consider marginal contributions: $\{2,3\}$ does not create worth but together with 1 it does. Hence, it has to be considered to assign the right distribution even though it ends up in a winning coalition not being minimal.
} 


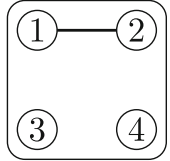

$g^{1-2}$

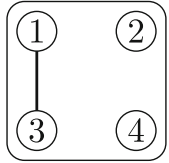

$g^{1-3}$



$g^{3-2-4}$

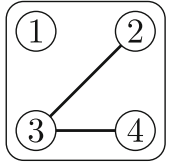

$g^{2-3-4}$

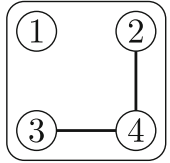

$g^{2-4-3}$

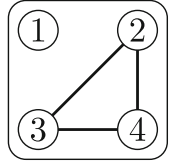

$g^{2-3-4-2}$

Fig. 1 Networks for minimal winning coalitions

Table 1 Payoffs

\begin{tabular}{lllllllllllll}
\hline & $\pi_{1}$ & $\pi_{2}$ & $\pi_{3}$ & $\pi_{4}$ & $\chi_{1}^{\#}$ & $\chi_{2}^{\#}$ & $\chi_{3}^{\#}$ & $\chi_{4}^{\#}$ & $\kappa_{1}$ & $\kappa_{2}$ & $\kappa_{3}$ & $\kappa_{4}$ \\
\hline$g^{1-2}$ & 0.5 & 0.5 & 0 & 0 & 0.58 & 0.42 & 0 & 0 & 0.56 & 0.44 & 0 & 0 \\
$g^{1-3}$ & 0.5 & 0 & 0.5 & 0 & 0.58 & 0 & 0.42 & 0 & 0.56 & 0 & 0.44 & 0 \\
$g^{3-2-4}$ & 0 & 0.5 & 0.25 & 0.25 & 0 & 0.39 & 0.39 & 0.22 & 0 & 0.42 & 0.35 & 0.23 \\
$g^{2-3-4}$ & 0 & 0.25 & 0.5 & 0.25 & 0 & 0.39 & 0.39 & 0.22 & 0 & 0.35 & 0.42 & 0.23 \\
$g^{2-4-3}$ & 0 & 0.25 & 0.25 & 0.5 & 0 & 0.39 & 0.39 & 0.22 & 0 & 0.36 & 0.36 & 0.28 \\
$g^{2-3-4-2}$ & 0 & 0.33 & 0.33 & 0.33 & 0 & 0.39 & 0.39 & 0.22 & 0 & 0.37 & 0.37 & 0.27 \\
\hline
\end{tabular}

second case while the Position value accounts for the different positions. On the other hand, the Position value does not take into account the existence of alternative winning coalitions, that is, outside options: consider the network $g^{3-2-4}$ again and note that agents 3 and 4 obtain the same payoff even though 3 would have the outside option of a winning coalition with 1 while 4 has no outside option. The graph- $\chi$ value accounts for outside options and assigns a higher payoff to agent 3 . The kappavalue takes into account both outside options and the position within the network: consider the coalition $\{2,3,4\}$, agents 2 and 3 always obtain a higher payoff than 4 , because they could also cooperate with 1 instead, while 4 has no outside option. Agents 2 and 3 are symmetric in terms of outside options but 2 obtains a higher payoff than 3 if the coalition is connected through 2, a lower payoff than 3 if the coalition is connected through 3 and the same payoff if the coalition is connected through 4. An agent obtains the highest payoff within the coalition if she has the strongest position.

Note that not every outside option has the same value/impact: consider $g^{1-2}$ and note that agents 1 and 2 have the same position in this network. Agent 1 obtains a higher payoff due to the fact that her outside option (building a coalition with 3 ) is valued higher/has a larger impact than the outside option of 2 (cooperating with $\{3,4\}$ ). This can be explained by the fact that in smaller coalitions, the worth has to be distributed among less agents.

In contrast to defining a coalition as the group of agents that is connected via path, one could define a coalition by complete subnetworks, that is, as the group of agents that are directly connected to each other. The latter definition has the drawback of not taking into account different interaction paths within a coalition and the position of all agents within the coalition would be the same. This is why we use the more general definition of a coalition. But still it is notable that also in the case of equal 
positions, the kappa-value is not redundant; it differs from the graph- $\chi$-value as shown by $g^{1-2}, g^{1-3}$ and $g^{2-3-4-2}$.

The paper is structured as follows: we start with the framework and drawbacks of the existing allocation rules. Following the idea of combining the properties of the analyzed allocation rules, Sect. 3 will give an axiomatic characterization of the kappa-value and analyzes independence of the characterizing axioms. Section 4 concludes.

\section{Framework and drawbacks}

\subsection{Framework}

For the framework, we follow Jackson and Wolinsky (1996). We model a network as an undirected graph where the nodes represent the players and the edges represent the links between the players. Let $N=\{1, \ldots, n\}$, non-empty and finite, be the player set. The complete network (in which there exists a link between any two players) is defined as $g^{N}:=\{i j:=\{i, j\} \mid i, j \in N\}$. Given that, we can define the set of all possible networks on $N: G_{N}:=\left\{g \mid g \subseteq g^{N}\right\}$.

A game in characteristic function form (or TU game $\left.{ }^{4}\right)(N, v)$ consists of the player set $N$ and a value function $v \in \mathbb{V}(N):=\left\{v: 2^{N} \longrightarrow \mathbb{R} \mid v(\emptyset)=0\right\}$, assigning to every $K \subseteq N$ the worth of the coalition $K . \mathbb{V}_{0}(N)$ is the set of all zero-normalized value functions, that is, if $v(\{i\})=0 \forall i \in N$. Without loss of generality, we will restrict ourselves to zero-normalized games since any $v \in \mathbb{V}(N)$ can be zero-normalized. An allocation rule $Y: N \times \mathbb{V}(N) \times G_{N} \longrightarrow \mathbb{R}^{N}$ distributes the arising worth among the players, that is, assigns a payoff to each player. An allocation rule is feasible if $\sum_{i \in N} Y_{i}(N, v, g) \leq v(N)$.

A very popular allocation rule is the Shapley value (Shapley 1953) ${ }^{5}$ :

$$
\begin{aligned}
\operatorname{Sh}_{i}(N, v) & :=\sum_{K \subseteq N \backslash\{i\}} \frac{k !(n-1-k) !}{n !}[v(K \cup\{i\})-v(K)], \\
\text { where } k & =|K|, n=|N| .
\end{aligned}
$$

The Shapley value assigns to every player her share of what she creates when entering a coalition, that is, her marginal contributions $[v(K \cup\{i\})-v(K)]$.

We say that players $i$ and $j$ are connected in the network $g$ if there exists a path $i i_{1}, i_{1} i_{2}, \ldots, i_{k} j \in g, i_{1}, \ldots, i_{k} \in N$. Connected players form components of a network $g$ and these components build a partition on the player set $N$. We denote this partition by $\mathcal{C}(N, g) ; C_{i}:=C_{i}(N, g) \in \mathcal{C}(N, g)$ is the component of all players connected with player $i \in N$.

\footnotetext{
4 Game with transferable utility.

5 The Shapley value is not restricted to network structures.
} 
Fig. 2 Train connections

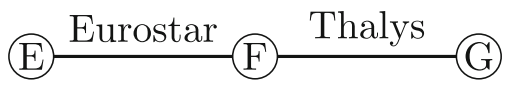

Myerson (1977) introduces the graph-restricted game

$$
\begin{aligned}
& v^{g}(K):=\sum_{S \in \mathcal{C}\left(K,\left.g\right|_{K}\right)} v(S), \\
& \quad \text { where }\left.g\right|_{K}=\{i j \in g \mid i, j \in K\}
\end{aligned}
$$

to define and characterize, as one of the first and most popular allocation rules for network structures, the Myerson value ${ }^{6}$ :

$$
\mu(N, v, g):=\operatorname{Sh}_{i}\left(N, v^{g}\right) .
$$

To define another popular allocation rule, Meessen (1988) and Borm et al. (1992) assign to every cooperation structure $(N, v, g)$ the link-game (or arc game) in which the links in the network are the players:

$$
v^{N}(g):=v^{g}(N)
$$

Recall that we restricted ourselves to zero-normalized games, otherwise $v^{N}$ might not be a value function because $v^{N}(\emptyset)=v^{\emptyset}(N) \neq 0$. Using the arc game, they introduce the Position value (for zero-normalized games):

$$
\begin{aligned}
& \pi_{i}(N, v, g):=\sum_{\lambda \in g_{i}} \frac{1}{2} \operatorname{Sh}_{\lambda}\left(g, v^{N}\right), \\
& \text { where } g_{i}=\text { set of links including player } i .
\end{aligned}
$$

The Position value takes into account the role of links in which a player is (directly) involved. For example: let $N=\{$ England (E), France (F), Germany (G) $\}$ and let $v(K)=1$ if $K=N$ and $v(K)=0$ otherwise. Consider the structure described by Fig. 2; England and France are connected by the Eurostar train, France and Germany are connected by the Thalys train, there is no train connection between England and Germany. The links are Eurostar $:=\{\mathrm{EF}\}$ and Thalys $:=\{\mathrm{FG}\}$. Both links are needed to create worth and Eurostar is needed as much as Thalys, that is, they are symmetric in the link-game. Hence, they both obtain half of the worth: $\mathrm{Sh}_{\text {Eurostar }}=\mathrm{Sh}_{\text {Thalys }}=\frac{1}{2}$. The Position value assigns to every player half of the payoff of every link she is involved in, that is, $\pi_{E}=\frac{1}{2} \mathrm{Sh}_{\text {Eurostar }}=\frac{1}{4}, \pi_{F}=\frac{1}{2} \mathrm{Sh}_{\text {Eurostar }}+\frac{1}{2} \mathrm{Sh}_{\text {Thalys }}=\frac{1}{2}$ and $\pi_{G}=\frac{1}{2} \mathrm{Sh}_{\text {Thalys }}=\frac{1}{4}$. The interpretation is that France is "needed more" to create the worth because it connects the three countries (transit country). Its position in the network is stronger which comes through the fact that it is involved in more links than the other two countries. Hence, it obtains a higher payoff.

\footnotetext{
6 Which is generalized by the equal bargaining rule (Jackson and Wolinsky 1996) to a wider class of networks.
} 




$g$

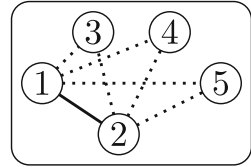

$g(1)=g(2)$

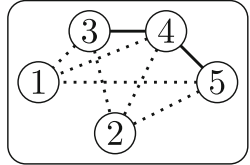

$g(3)=g(4)=g(5)$

Fig. 3 Network and corresponding LOOGs

Casajus (2009a) introduces an outside-option-sensitive allocation rule. To reflect all (productive) outside options, he defines for every network $g$ the corresponding lower outside-option graph ( $L O O G)$, the graph that captures the alternatives/outside options the players have: $g(i, N):=\left.g\right|_{C_{i}} \cup\left\{j k \in g^{N} \mid j \in C_{i}, k \in N \backslash C_{i}\right\}$. For notational convenience, if the player set is fixed, we will only write $g(i)$. Consider the following example: let $N=\{1,2,3,4,5\}$ and $g=\{12,34,45\}$, the LOOGs are displayed in Fig. 3. The LOOG reflects all alternative links a player and her coalitional players might have outside their actual coalition (which stays fixed). To provide these alternatives, links outside the own coalition are broken.

Using the LOOG, Casajus (2009a) defines the graph- $\chi$-value:

$$
\begin{aligned}
\chi_{i}^{\#}(N, v, g) & :=\mu_{i}(N, v, g(i))+\frac{v\left(C_{i}\right)-\mu_{C_{i}}(N, v, g(i))}{\left|C_{i}\right|}, \\
\text { where } \varphi_{K} & =\sum_{i \in K} \varphi_{i}
\end{aligned}
$$

The graph- $\chi$-value values outside options by adding/substracting some share of the Myerson value of her component to the Myerson value of the player (where the Myerson value is the Shapley value of the graph-restricted game), taking into account the outside option graph.

\subsection{Drawbacks of existing allocation rules}

The Position value and the Myerson value (as well the equal bargaining rule) are component decomposable, that is, we can restrict the game to components. Component Decomposability states that the player's outside world does not affect payoffs within a component. Hence, it stands in contradiction to outside-option-sensitivity (Belau 2011). That is, both the Position value and the Myerson value (as well as the equal bargaining rule) generally do not account for outside options.

On the other hand, one can show that the Myerson value (as well as the equal bargaining rule) and the graph- $\chi$-value do not always take into account the specific position of a player within the network (i.e., the communication path). This holds, for example, for weighted voting games as the one given in the introduction. ${ }^{7}$

\footnotetext{
7 Proofs and further information can be requested from the author.
} 
Hence, to account for both outside options and the position of a player within the network, we need a new allocation rule.

\section{A new outside option value: the kappa-value}

In this section, we define a new allocation rule for network structures which takes into account both outside options and the position of an agent within the network/the path of information flow. One can show that (for a large class of games) the graph- $\chi$ value does not differ within the class of networks referring to the same coalition. On the other hand, it takes outside options into account. While the latter is not true for the Position value, it takes the position of an agent within a network into account. We can show that the Myerson value lacks both desired properties. Hence, we will further analyze the characterizing axioms of the graph- $\chi$-value and the Position value.

An allocation rule satisfies Component Efficiency $(C E)$ if for all $C \in \mathcal{C}(N, g)$ we have

$$
\sum_{i \in C} Y_{i}(N, v, g)=v(C)
$$

Here, we interpret the components as productive unions. A productive union should get the payoff it creates. An allocation rule satisfies Balanced Link Contributions (BLC) if for all $i, j \in N, i \neq j$ and $v \in \mathbb{V}_{0}$ we have

$$
\sum_{\lambda \in g_{j}}\left[Y_{i}(N, v, g)-Y_{i}(N, v, g-\lambda)\right]=\sum_{\lambda \in g_{i}}\left[Y_{j}(N, v, g)-Y_{j}(N, v, g-\lambda)\right]
$$

Slikker (2005) argues that BLC "deals with the loss players can inflict on each other. The total threat of a player towards another player is defined as the sum over all links of the first player of the payoff differences the second player experiences if such a link is broken." BLC states that the total threat of a player towards another player should be equal to the reverse total threat. Slikker (2005) shows that the Position value is characterized by CE and BLC.

We note that the Position value also satisfies the following axiom: an allocation rule satisfies Component Decomposability $(C D)$ if for all $i \in C \in \mathcal{C}(N, g)$ we have $Y_{i}(N, v, g)=Y_{i}\left(C,\left.v\right|_{C},\left.g\right|_{C}\right)$. CD stands in contradiction to outside-optionsensitivity (cf. Sect. 2.2). Hence, if we want an allocation rule to account for outside options but still to take into account the role of a player within the network, we need to weaken BLC (in order to get rid of the allocation rule satisfying CD but still having $\mathrm{CE})$.

On connected graphs, outside options are not present. In the following, we give an axiom which captures the idea of $\mathrm{BLC}^{8}$ in situations where outside options are absent:

\footnotetext{
8 Or the axiom "equal bargaining power" in the Jackson and Wolinsky-sense.
} 
Definition 1 (Weak Balanced Link Contributions (WBLC)) For all connected $g$ and all $i, j \in N$ and $v \in \mathbb{V}_{0}$ we have

$$
\begin{aligned}
& \sum_{\lambda \in g_{j}}\left[Y_{i}(N, v, g)-Y_{i}\left(C_{i}(N, g-\lambda),\left.v\right|_{C_{i}(N, g-\lambda)},\left.g\right|_{C_{i}(N, g-\lambda)}\right)\right] \\
& =\sum_{\lambda \in g_{i}}\left[Y_{j}(N, v, g)-Y_{j}\left(C_{j}(N, g-\lambda),\left.v\right|_{C_{j}(N, g-\lambda)},\left.g\right|_{C_{j}(N, g-\lambda)}\right)\right] .
\end{aligned}
$$

Following the idea of BLC, WBLC also equalizes threats. In contrast to equalizing total threats as defined by Slikker (2005), WBLC compares payoff differences experienced if not only a link is broken but rather the whole situation is reduced to what is left in a coalition after a link is broken (restriction to components). We restrict to components because within connected components, again, outside options are absent. ${ }^{9}$ Note that in presence of CD, WBLC reduces to BLC on connected graphs.

Lemma 1 If $Y$ satisfies $C E$ and WBLC, it coincides with the Position value for all connected graphs.

Find the proof in the Appendix.

If we combine Lemma 1 with the presence of $\mathrm{CD}$, we will have a characterization of the Position value. Hence, we need to weaken CD. We use the characterizing axiom of the graph- $\chi$-value that accounts for outside options given by Casajus (2009a): an allocation rule satisfies Outside Option Consistency $(O O)$ if for all $i, j \in C \in \mathcal{C}(N, g)$ we have

$$
Y_{i}(N, v, g)-Y_{j}(N, v, g)=Y_{i}(N, v, g(i))-Y_{j}(N, v, g(j)) .
$$

Theorem 1 (The kappa-value) Let $v \in \mathbb{V}_{0}$. There is a unique allocation rule for network structures that satisfies CE, $O O$ and WBLC:

$$
\kappa_{i}(N, v, g):=\pi_{i}(N, v, g(i))+\frac{v\left(C_{i}\right)-\pi_{C_{i}}(N, v, g(i))}{\left|C_{i}\right|}
$$

Proof We follow the idea of the uniqueness proof of the graph- $\chi$-value (Casajus 2009a). Let $Y$ satisfy CE, OO and WBLC. For $i, j \in C_{i}$ we have $g(i)=g(j)$. By OO:

$$
Y_{i}(N, v, g)-Y_{i}(N, v, g(i))=Y_{j}(N, v, g)-Y_{j}(N, v, g(i))
$$

Summing up over $j \in C_{i}$ and using CE gives

$$
\left|C_{i}\right|\left[Y_{i}(N, v, g)-Y_{i}(N, v, g(i))\right]=v\left(C_{i}\right)-Y_{C_{i}}(N, v, g(i))
$$

\footnotetext{
9 WBLC combines the ideas underlying BLC and Weak Fairness 2 (Casajus 2009a), where the latter is a modification of the fairness property employed by Myerson (1977) to characterize the Myerson value.
} 
Since $g(i)$ is connected, Lemma 1 implies $Y_{i}(N, v, g(i))=\pi_{i}(N, v, g(i))$ for all $i \in N$ and hence we have

$$
Y_{i}(N, v, g)=\pi_{i}(N, v, g(i))+\frac{v\left(C_{i}\right)-\pi_{C_{i}}(N, v, g(i))}{\left|C_{i}\right|}
$$

which uniquely determines $Y$.

It is easily shown that the value given by Eq. (1) satisfies CE and OO (note that $(g(i))(i)=g(i))$. WBLC follows after some calculations (see Appendix).

We call the value given by (1) "kappa-value" and denote it by $\kappa$. The kappa-value assigns to each player in a coalition the worth of the position she would obtain in the outside option graph plus her share of the worth players outside the actual coalition would obtain in the outside option graph. This share is equal for all players in the actual coalition and the worth of a position is given by the Shapley value of the arc game, the Position value.

The kappa-value provides a very elegant use of the quite intuitive Position value, lacking its drawbacks by using the outside option graph. Note that $\pi(N, v, g(i))$ also captures both positions in the network and outside options, but it is not feasible: It might be the case that $\sum_{i \in N} \pi_{i}(N, v, g(i))>v(N)$ since the LOOG differs for agents in different coalitions. ${ }^{10}$ In order to avoid the mentioned drawbacks of the graph- $\chi$ value, the kappa-value differs by using the Position value instead of the Myerson value. Note that the Myerson value of the LOOG $(\mu(N, v, g(i)))$ also has the drawback of not always accounting for the position of an agent within the network. ${ }^{11}$ Hence, there is no simpler alternative to the kappa-value.

We now show that the axioms CE, OO and WBLC are independent. Consider the weighted voting game from the introduction $(N=\{1,2,3,4\}, w=(39,30,25,6)$ and $T=60)$.

Independence 1 (CE+WBLC, $\neg \mathrm{OO})$ The Position value satisfies $C E$ and WBLC (because of BLC and CD). Consider $g=\{12\}$. We have

$$
\begin{aligned}
& \pi_{1}(N, v, g)-\pi_{2}(N, v, g)=\frac{1}{2}-\frac{1}{2}=0 \text { and } \\
& \pi_{1}(N, v, g(1))-\pi_{2}(N, v, g(2))=\frac{49}{120}-\frac{34}{120} \neq 0
\end{aligned}
$$

$\Rightarrow$ OO is violated.

\footnotetext{
10 Consider the example from the introduction and $g=\{23,24,34\}$, then $g(2)=g(3)=g(4)$ while $g(1)$ differs and $\pi_{1}(N, v, g(1))=1 / 2>2 / 5=\pi_{1}(N, v, g(2))$, hence $\sum_{i \in N} \pi_{i}(N, v, g(i))>$ $\sum_{i \in N} \pi_{i}(N, v, g(2))=v(N)$ (by $\pi$ satisfying CE and $C_{i}=N$ on $g(i)$ ).

11 Further information can be requested by the author.
} 
Independence 2 (CE+OO, $\neg$ WBLC) The graph- $\chi$-value satisfies $C E$ and OO. Consider $g=\{12,23,34\}$. We have

$$
\begin{aligned}
& \sum_{\lambda \in g_{1}}\left[\chi_{2}^{\#}(N, v, g)-\chi_{2}^{\#}\left(C_{2}(N, g-\lambda),\left.v\right|_{C_{2}(N, g-\lambda)},\left.g\right|_{C_{2}(N, g-\lambda)}\right)\right] \\
& \quad=\chi_{2}^{\#}(N, v, g)-\chi_{2}^{\#}\left(N \backslash\{1\},\left.v\right|_{N \backslash\{1\}}, g-12\right)=\frac{7}{12}-\frac{1}{6}=\frac{5}{12} \\
& \text { and } \\
& \sum_{\lambda \in g_{2}}\left[\chi_{1}^{\#}(N, v, g)-\chi_{1}^{\#}\left(C_{1}(N, g-\lambda),\left.v\right|_{C_{1}(N, g-\lambda)},\left.g\right|_{C_{1}(N, g-\lambda)}\right)\right] \\
& =2 \chi_{1}^{\#}(N, v, g)-\chi_{1}^{\#}\left(\{1\},\left.v\right|_{\{1\}}, \emptyset\right)-\chi_{1}^{\#}\left(\{1,2\},\left.v\right|_{\{1,2\}},\{12\}\right) \\
& =2 \cdot \frac{3}{12}-0-\frac{1}{2}=0 \neq \frac{5}{12}
\end{aligned}
$$

$\Rightarrow W B L C$ is violated.

Now consider the TU-game $(N, v)$ with $N=\{1,2,3,4\}$ and $v=u_{N}$.

Independence 3 (WBLC+OO, $\neg \mathrm{CE}) Y_{i}(N, v, g):=\pi_{i}(N, v, g(i))$ satisfies $O O$ by $(g(i))(i)=g(i)$ and $W B L C$ by $Y=\pi$ for connected graphs. Consider $g=\{12,34\}$. We have

$$
\begin{gathered}
Y_{1}(N, v, g)=Y_{3}(N, v, g)=\frac{13}{60}, Y_{2}(N, v, g)=\frac{17}{60} \text { and } Y_{4}(N, v, g)=\frac{1}{2} \\
\Rightarrow Y_{C_{4}}=Y_{4}=\frac{1}{2} \neq 0=u_{N}(\{4\}) \text { and } Y_{\{1,2,3\}}=\frac{43}{60} \neq 0=u_{N}(\{1,2,3\})
\end{gathered}
$$

$\Rightarrow C E$ is violated.

\section{Conclusion and further research}

We analyzed the Position value, which takes the position of a player within the network into account, and the graph- $\chi$-value, which takes outside options into account. The graph- $\chi$-value does not always account for the position an agent has within the network, this motivated the use of the Position value in order to capture the fact that the position within the network/the path of information flow matters. But the Position value generally does not account for outside options. We defined and characterized a new allocation rule for networks which combines outside-option-sensitivity and sensitivity to the position of an agent within the network: the kappa-value. The kappavalue provides an elegant use of the intuitive concept of the Position value, lacking its drawbacks by using the outside option graph used for the concept of the graph$\chi$-value. There is no need for new characterizing axioms, only known and approved ones (or weakened versions of them) are used.

On the application point of view, it is not sure whether outside-option-sensitivity is always desirable, since it might not always be meaningful to consider outside options. 
As mentioned, experimental and theoretical works show that outside options do affect bargaining. As an application one could consider trade agreements: there exist different possible trade routes (outside options) and furthermore, transit countries and other countries should be treated differently (position within the network). Using allocation rules, we can distribute the worth of the output good among the countries. This can also be seen as measuring the (relative) distribution of power of the countries. As other applications where outside options matter one could consider defense agreements (taking into account rights of way) or political agreements: there is a specific vote distribution among parties in a parliament and the parties have to build agreements in order to reach some required quorum (for example, to pass a bill). Here, we can use allocation rules to measure the (relative) distribution of power of the parties.

For future work, we plan to analyze the performance of the kappa-value in a trade model as well as an analysis of stability under the kappa-value; for example, analyzing pairwise stability (Jackson and Wolinsky 1996).

Acknowledgments I want to thank Jörg Franke, Sebastian Garmann, Jan Heufer, Wolfgang Leininger, Lars Metzger and Andrew Monaco for comments and suggestions. Financial support by the Ruhr Graduate School in Economics is gratefully acknowledged.

\section{Appendix}

\section{Proof of Lemma 1}

Proof Note that in presence of CD, WBLC reduces to BLC on connected graphs. Hence, since the Position value satisfies $\mathrm{CD}$ and is characterized by $\mathrm{CE}$ and BLC, it satisfies CE and WBLC (which provides existence). For uniqueness, we cannot use the presence of CD any longer, hence, we cannot use BLC. We follow the idea of the proof for the Myerson value of Casajus (2009a). Let $\varphi$ and $\psi$ satisfy CE and WBLC. Suppose $N$ is the minimal player set such that $\varphi$ and $\psi$ differ on a connected graph. We must have $|N|>1$, because for $|N|=1$ we would have a contradiction by CE due to the connectedness of the graph. Suppose that $g$ is the minimal connected graph on $N$ such that $\varphi \neq \psi$.

Let $i, j \in N$. By WBLC we have

$$
\begin{aligned}
& \left|g_{j}\right| \varphi_{i}(N, v, g)-\left|g_{i}\right| \varphi_{j}(N, v, g)=\sum_{\lambda \in g_{j}} \varphi_{i}\left(C_{i}(N, g-\lambda),\left.v\right|_{C_{i}(N, g-\lambda)},\left.g\right|_{C_{i}(N, g-\lambda)}\right) \\
& \quad-\sum_{\lambda \in g_{i}} \varphi_{j}\left(C_{j}(N, g-\lambda),\left.v\right|_{C_{j}(N, g-\lambda)},\left.g\right|_{C_{j}(N, g-\lambda)}\right)
\end{aligned}
$$

Note that $\left.g\right|_{C}$ is always connected on the connected component $C$. If now $C_{k}(N, g-$ $\lambda) \neq N$ (for $k=i, j)$, we have $C_{k}(N, g-\lambda) \subset N$ and hence $\varphi_{k}\left(C_{k}(N, g-\right.$ $\left.\lambda),\left.v\right|_{C_{k}(N, g-\lambda)},\left.g\right|_{C_{k}(N, g-\lambda)}\right)=\psi_{k}\left(C_{k}(N, g-\lambda),\left.v\right|_{C_{k}(N, g-\lambda)},\left.g\right|_{C_{k}(N, g-\lambda)}\right)$ since $N$ is the minimal player such that $\varphi$ and $\psi$ differ on a connected graph. If $C_{k}(N, g-\lambda)=N$ we have that $g-\lambda$ is connected on $N$ and hence $\varphi_{k}\left(C_{k}(N, g-\right.$ 
$\left.\lambda),\left.v\right|_{C_{k}(N, g-\lambda)},\left.g\right|_{C_{k}(N, g-\lambda)}\right)=\psi_{k}\left(C_{k}(N, g-\lambda),\left.v\right|_{C_{k}(N, g-\lambda)},\left.g\right|_{C_{k}(N, g-\lambda)}\right)$ since $g$ is the minimal connected graph on $N$ such that $\varphi$ and $\psi$ differ.

Using that $\psi$ satisfies WBLC, we get

$$
\begin{aligned}
& \left|g_{j}\right| \varphi_{i}(N, v, g)-\left|g_{i}\right| \varphi_{j}(N, v, g)=\left|g_{j}\right| \psi_{i}(N, v, g)-\left|g_{i}\right| \psi_{j}(N, v, g) \\
& \quad \Leftrightarrow\left|g_{j}\right|\left[\varphi_{i}(N, v, g)-\psi_{i}(N, v, g)\right]=\left|g_{i}\right|\left[\varphi_{j}(N, v, g)-\psi_{j}(N, v, g)\right]
\end{aligned}
$$

Summing up over $j \in C_{i}(N, g)=N$ (connected graph), we have by CE:



and hence $\varphi_{i}(N, v, g)=\psi_{i}(N, v, g)$.

\section{Proof of WBLC in Theorem 1}

Proof To see WBLC, first note that the Position value satisfies WBLC by satisfying BLC and CD. Let $g$ be connected and $i, j \in N$, then we have $C_{i}=C_{j}=N$ and $g(i)=g(j)=g$. Hence,

$$
\begin{aligned}
& \sum_{\lambda \in g_{j}} Y_{i}(N, v, g)-\sum_{\lambda \in g_{j}} Y_{j}(N, v, g) \\
& =\sum_{\lambda \in g_{j}}[\pi_{i}(N, v, g)+\underbrace{\frac{v(N)-\pi_{N}(N, v, g)}{|N|}}_{=0 \text { by CE }}] \\
& -\sum_{\lambda \in g_{i}}[\pi_{j}(N, v, g)+\underbrace{\frac{v(N)-\pi_{N}(N, v, g)}{|N|}}_{=0 \text { by } \mathrm{CE}}] \\
& =\sum_{\lambda \in g_{j}} \pi_{i}(N, v, g)-\sum_{\lambda \in g_{i}} \pi_{j}(N, v, g) \\
& \stackrel{\mathrm{WBLC}}{=} \sum_{\lambda \in g_{j}} \pi_{i}\left(C_{i}(N, g-\lambda),\left.v\right|_{C_{i}(N, g-\lambda)},\left.g\right|_{C_{i}(N, g-\lambda)}\right) \\
& -\sum_{\lambda \in g_{j}} \pi_{j}\left(C_{j}(N, g-\lambda),\left.v\right|_{C_{j}(N, g-\lambda)},\left.g\right|_{C_{j}(N, g-\lambda)}\right)
\end{aligned}
$$




$$
\begin{aligned}
= & \sum_{\lambda \in g_{j}} Y_{i}\left(C_{i}(N, g-\lambda),\left.v\right|_{C_{i}(N, g-\lambda)},\left.g\right|_{C_{i}(N, g-\lambda)}\right) \\
& -\sum_{\lambda \in g_{j}} Y_{j}\left(C_{j}(N, g-\lambda),\left.v\right|_{C_{j}(N, g-\lambda)},\left.g\right|_{C_{j}(N, g-\lambda)}\right)
\end{aligned}
$$

where the last step drops from the fact that $\left.g\right|_{C_{k}(N, g-\lambda)}$ is connected on $C_{k}(N, g-\lambda)$ and by $\mathrm{CE}$.

\section{References}

Aumann, R., Drèze, J.: Cooperative games with coalition structures. Int. J. Game Theory 3(4), 217-237 (1974)

Belau, J.: Outside options in probabilistic coalition situations. Int. Game Theory Rev. 13(4), 417-442 (2011)

Borm, P., Owen, G., Tijs, S.: On the position value for communication situations. SIAM J. Discrete Math. 5, 305-320 (1992)

Casajus, A.: Networks and outside options. Soc. Choice Welf. 32, 1-13 (2009a)

Casajus, A.: Outside options, component efficiency and stability. Games Econ. Behav. 65, 49-61 (2009b)

González-Arangüena, E., Manuel, C., Gómez, D., van den Brink, R.: A value for directed communication situations. Tinbergen Institute Discussion Paper 8-006/1 (2008)

Jackson, M., Wolinsky, A.: A strategic model of social and economic networks. J. Econ. Theory 71, 44-74 (1996)

Maschler, M.: The bargaining set, kernel, and nucleolus. In: Aumann, R.J., Hart, S. (eds.) Handbook of Game Theory with Economic Applications, vol. I, Chap. 34, pp. 591-667. Elsevier, Amsterdam (1992)

Meessen, R.: Communication games. Department of Mathematics, University of Nijmegen, The Netherlands, Master's Thesis (1988)

Myerson, R.: Graphs and cooperations in games. Math. Oper. Res. 2, 225-229 (1977)

Nowak, A., Radzik, T.: The shapley value for $\mathrm{n}$-person games in generalized characteristic function form. Games Econ. Behav. 6, 150-161 (1994)

Owen, G.: Values of games with a priori unions. In: Henn, R., Moeschlin, O. (eds.) Mathematical Economics and Game Theory, pp. 76-87. Springer, Berlin (1977)

Shapley, L.: A value for n-person games. In: Kuhn, H., Tucker, A. (eds.) Contributions to the Theory of Games, vol. II, pp. 307-317. Princeton University Press, Princeton (1953)

Shapley, L., Shubik, M.: Pure competition, coalitional power and fair division. Int. Econ. Rev. 10(3), 337362 (1969)

Slikker, M.: A characterization of the position value. Int. J. Game Theory 33(4), 504-514 (2005)

Tutic, A., Pfau, S., Casajus, A.: Experiments on bilateral bargaining in markets. Theory Decis. 70(4), 529-546 (2011)

van den Brink, R., van der Laan, G., Vasil'ev, V.: Component efficient solutions in line-graph games with applications. Econ. Theory 33(2), 349-364 (2007)

van den Brink, R., Katsev, I., van der Laan, G.: Axiomatizations of two types of Shapley values for games on union closed systems. Econ. Theory 47(1), 175-188 (2011)

von Neumann, J., Morgenstern, O.: Theory of games and economic behavior. Princeton University Press, Princeton (1944)

Wiese, H.: Measuring the power of parties within government coalitions. Int. Game Theory Rev. 9, 307-322 (2007) 